\title{
Three-Dimensional Grid Drawings with Sub-quadratic Volume ${ }^{\star}$
}

\author{
Vida Dujmović ${ }^{1}$ and David R. Wood $^{2}$ \\ 1 School of Computer Science, McGill University, Montréal, Canada \\ vida@cs.mcgill.ca \\ 2 School of Computer Science, Carleton University, Ottawa, Canada \\ davidw@scs. carleton.ca
}

\begin{abstract}
A three-dimensional grid drawing of a graph is a placement of the vertices at distinct points with integer coordinates, such that the straight line-segments representing the edges are pairwise non-crossing. A $\mathcal{O}\left(n^{3 / 2}\right)$ volume bound is proved for three-dimensional grid drawings of graphs with bounded degree, graphs with bounded genus, and graphs with no bounded complete graph as a minor. The previous best bound for these graph families was $\mathcal{O}\left(n^{2}\right)$. These results (partially) solve open problems due to Pach, Thiele, and Tóth [Graph Drawing 1997] and Felsner, Liotta, and Wismath [Graph Drawing 2001].
\end{abstract}

\section{Introduction}

A three-dimensional straight-line grid drawing of a graph, henceforth called a $3 D$ drawing, is a placement of the vertices at distinct points in $\mathbb{Z}^{3}$ (called gridpoints), such that the line-segments representing the edges are pairwise noncrossing. That is, distinct edges only intersect at common endpoints, and each edge only intersects a vertex that is an endpoint of that edge. In contrast to the case in the plane, it is well known that every graph has a $3 \mathrm{D}$ drawing. We are therefore interested in optimising certain measures of the aesthetic quality of such drawings.

The bounding box of a 3D drawing is the minimum axis-aligned box containing the drawing. If the bounding box has side lengths $X-1, Y-1$ and $Z-1$, then we speak of an $X \times Y \times Z$ drawing with volume $X \cdot Y \cdot Z$. That is, the volume of a 3D drawing is the number of gridpoints in the bounding box. This definition is formulated so that $2 \mathrm{D}$ drawings have positive volume. We are interested in $3 \mathrm{D}$ drawings with small volume, which is a widely studied problem $3,4,5] 6] 7,8,10$, 1112 14 18 24 25127. Three-dimensional graph drawings in which the vertices are allowed real coordinates have also been studied (see the references in 11]). The authors have also established bounds on the volume of three-dimensional polyline grid drawings, where bends in the edges are also at gridpoints 11. Table 1 summarises the best known upper bounds on the volume of 3D drawings, including those established in this paper.

\footnotetext{
* Research supported by NSERC and FCAR. 
Table 1. Upper bounds on the volume of 3D drawings of $n$-vertex $m$-edge graphs.

\begin{tabular}{lll}
\hline graph family & volume & reference \\
\hline arbitrary & $\mathcal{O}\left(n^{3}\right)$ & Cohen et al. [5] \\
arbitrary & $\mathcal{O}\left(m^{4 / 3} n\right)$ & Theorem 4] \\
maximum degree $\Delta$ & $\mathcal{O}(\Delta m n)$ & Theorem 3] \\
constant maximum degree & $\mathcal{O}\left(n^{3 / 2}\right)$ & Theorem[10] \\
constant chromatic number & $\mathcal{O}\left(n^{2}\right)$ & Pach et al. [24] \\
constant chromatic number & $\mathcal{O}\left(m^{2 / 3} n\right)$ & Theorem 6] \\
no $K_{h}$-minor $(h$ constant) & $\mathcal{O}\left(n^{3 / 2}\right)$ & Theorem [] \\
constant genus & $\mathcal{O}\left(n^{3 / 2}\right)$ & Theorem 8] \\
constant tree-width & $\mathcal{O}(n)$ & Dujmović and Wood [12] \\
\hline
\end{tabular}

Cohen et al. 5 proved that every graph has a 3D drawing with $\mathcal{O}\left(n^{3}\right)$ volume, and that this bound is asymptotically optimal for complete graphs $K_{n}$. Our edgesensitive bounds of $\mathcal{O}\left(m^{4 / 3} n\right)$ and $\mathcal{O}(\Delta m n)$ are greater than $\mathcal{O}\left(n^{3}\right)$ in the worst case. It is an open problem whether there are edge-sensitive bounds that match the $\mathcal{O}\left(n^{3}\right)$ bound in the case of complete graphs.

Pach et al. 24] proved that graphs with constant chromatic number have $3 \mathrm{D}$ drawings with $\mathcal{O}\left(n^{2}\right)$ volume. For $c$-colourable graphs the actual bound is $\mathcal{O}\left(c^{2} n^{2}\right)$. Our edge-sensitive bound of $\mathcal{O}\left(m^{2 / 3} n\right)$ is an improvement on this result for graphs with constant chromatic number and $o\left(n^{3 / 2}\right)$ edges. Pach et al. [24] also proved an $\Omega\left(n^{2}\right)$ lower bound for the volume of $3 \mathrm{D}$ drawings of complete bipartite graphs $K_{n, n}$. This lower bound was generalised to all graphs by Bose et al. [3, who proved that every 3D drawing has volume at least $\frac{1}{8}(n+m)$.

Graphs with constant maximum degree have constant chromatic number, and by the result of Pach et al. 24], have 3D drawings with $\mathcal{O}\left(n^{2}\right)$ volume. Pach et al. 24] conjectured that graphs with constant maximum degree have $3 \mathrm{D}$ drawings with $o\left(n^{2}\right)$ volume. We verify this conjecture by proving that such graphs have $3 \mathrm{D}$ drawings with $\mathcal{O}\left(n^{3 / 2}\right)$ volume.

The first $\mathcal{O}(n)$ upper bound on the volume of $3 \mathrm{D}$ drawings was established by Felsner et al. 14 for outerplanar graphs. This result was generalised by the authors for graphs with constant tree-width [12. Improved constants have been obtained in a number of special cases 6 6 1818. Felsner et al. 14] proposed the following inviting open problem: does every planar graph have a 3D drawing with $\mathcal{O}(n)$ volume? In this paper we provide a partial solution to this question, by proving that planar graphs have $3 \mathrm{D}$ drawings with $\mathcal{O}\left(n^{3 / 2}\right)$ volume. Note that $\mathcal{O}\left(n^{2}\right)$ is the optimal area for plane 2D grid drawings, and $\mathcal{O}\left(n^{2}\right)$ was the previous best upper bound on the volume of 3D drawings of planar graphs.

A graph $H$ is a minor of a graph $G$ if $H$ is isomorphic to a graph obtained from a subgraph of $G$ by contracting edges. The genus of a graph $G$ is the minimum $\gamma$ such that $G$ can be embedded in the orientable surface with $\gamma$ handles. Of course, planar graphs have genus 0 and no $K_{5}$-minor. A generalisation of our result for planar graphs is that every graph with constant genus or with no $K_{h}$-minor for constant $h$ has a $3 \mathrm{D}$ drawing with $\mathcal{O}\left(n^{3 / 2}\right)$ volume. 


\section{Track Layouts}

We consider undirected, finite, and simple graphs $G$ with vertex set $V(G)$ and edge set $E(G)$. The number of vertices and edges of $G$ are respectively denoted by $n=|V(G)|$ and $m=|E(G)|$. A vertex c-colouring of $G$ is a partition $\left\{V_{i}\right.$ : $1 \leq i \leq c\}$ of $V(G)$, such that for every edge $v w \in E(G)$, if $v \in V_{i}$ and $w \in V_{j}$ then $i \neq j$. Each $i \in\{1,2, \ldots, c\}$ is a colour, and each set $V_{i}$ is a colour class. At times it will be convenient to write $\operatorname{col}(v)=i$ rather than $v \in V_{i}$. If $G$ has a vertex $c$-colouring then $G$ is c-colourable. The chromatic number of $G$, denoted by $\chi(G)$, is the minimum $c$ such that $G$ is $c$-colourable.

Let $\left\{V_{i}: 1 \leq i \leq c\right\}$ be a vertex $c$-colouring of a graph $G$. Let $<_{i}$ be a total order on each colour class $V_{i}$. Then each pair $\left(V_{i},<_{i}\right)$ is a track, and $\left\{\left(V_{i},<_{i}\right): 1 \leq i \leq t\right\}$ is a $t$-track assignment of $G$. To ease the notation we denote track assignments by $\left\{V_{i}: 1 \leq i \leq c\right\}$ when the ordering on each colour class is implicit. An $X$-crossing in a track assignment consists of two edges $v w$ and $x y$ such that $v<_{i} x$ and $y<_{j} w$, for distinct colours $i$ and $j$. A $t$-track layout of $G$ consists of a $t$-track assignment of $G$ with no X-crossing. The track-number of $G$, denoted by $\operatorname{tn}(G)$, is the minimum $t$ such that $G$ has a $t$-track layout 1

Track layouts and track-number were introduced in [10]12] although they are implicit in many previous works 1419. Track layouts and 3D drawings are closely related, as illustrated by the following result by Dujmović et al. 10. Also note that other authors have used track layouts to produce 3D drawings 68, 18, and there is a tight relationship between track layouts and another type of graph layout called a queue layout [27, which is a dual structure to a book embedding introduced by Heath et al. [19].

Theorem 1. [10] Every n-vertex graph $G$ with track-number $\operatorname{tn}(G) \leq t$ has a $2 t \times 4 t \times 4 t\left\lceil\frac{n}{t}\right\rceil$ drawing, which has $\mathcal{O}\left(t^{2} n\right)$ volume. Conversely, if a graph $G$ has an $X \times Y \times Z$ drawing then $G$ has track-number $\operatorname{tn}(G) \leq 2 X Y$.

We have the following upper bounds on the track-number.

Lemma 1. Let $G$ be a graph with $n$ vertices, maximum degree $\Delta$, path-width $p$, tree-width $w$, genus $\gamma$, and with no $K_{h}$-minor. Then the track-number of $G$ satisfies: (a) $\operatorname{tn}(G) \leq p+1, \quad$ (b) $\operatorname{tn}(G) \leq \mathcal{O}\left(6^{4^{w}}\right), \quad$ (c) $\operatorname{tn}(G) \leq 72 w \Delta$, (d) $\operatorname{tn}(G) \in \mathcal{O}\left(\gamma^{1 / 2} n^{1 / 2}\right), \quad$ (e) $\operatorname{tn}(G) \in \mathcal{O}\left(h^{3 / 2} n^{1 / 2}\right)$.

Proof. Part (a) is by Dujmović et al. 10. Parts (b) and (c) are by the authors [12. Gilbert et al. [16] and Djidjev 9] independently proved that $G$ has a $\mathcal{O}\left(\gamma^{1 / 2} n^{1 / 2}\right)$-separator, and thus has $\mathcal{O}\left(\gamma^{1 / 2} n^{1 / 2}\right)$ path-width (see Bodlaender [2, Theorem 20(iii)]). Hence (d) follows from (a). Similarly (e) follows from the result by Alon et al. [1] that $G$ has a $\mathcal{O}\left(h^{3 / 2} n^{1 / 2}\right)$-separator.

The next result is the fundamental contribution of this section.

Theorem 2. Every graph $G$ with $m$ edges and maximum degree $\Delta$ has tracknumber $\operatorname{tn}(G) \leq 14 \sqrt{\Delta m}$.

1 This definition of track-number is unrelated to that of Gyárfás and West [17. 
To prove Theorem 2 we introduce the following concept. A vertex colouring is a strong star colouring if between every pair of colour classes, all edges (if any) are incident to a single vertex. That is, each bichromatic subgraph consists of a star and possibly some isolated vertices. The strong star chromatic number of a graph $G$, denoted by $\chi_{\text {sst }}(G)$, is the minimum number of colours in a strong star colouring of $G$. Note that star colourings, in which each bichromatic subgraph is a forest of stars, have also been studied (see 23 for example). The star chromatic number of a graph $G$, denoted by $\chi_{\text {st }}(G)$, is the minimum number of colours in a star-colouring of $G$. With an arbitrary order on each colour class in a strong star colouring, there is no X-crossing. Thus track-number $\operatorname{tn}(G) \leq \chi_{\text {sst }}(G)$ for every graph $G$, and Theorem 2 is an immediate corollary of the next lemma.

Lemma 2. Every graph $G$ with $m$ edges and maximum degree $\Delta \geq 1$ has strong star chromatic number $\chi_{\mathrm{sst}}(G) \leq 14 \sqrt{\Delta m}$.

The proof of Lemma 2 uses the weighted version of the Lovász Local Lemma [13].

Lemma 3. [22, p. 221] Let $\mathcal{E}=\left\{A_{1}, \ldots, A_{n}\right\}$ be a set of 'bad' events. Let $0 \leq p \leq \frac{1}{4}$ be a real number, and let $t_{1}, \ldots, t_{n} \geq 1$ be integers. Suppose that for all $A_{i} \in \mathcal{E}$,

(a) the probability $\mathbf{P}\left(A_{i}\right) \leq p^{t_{i}}$,

(b) $A_{i}$ is mutually independent of $\mathcal{E} \backslash\left(\left\{A_{i}\right\} \cup D_{i}\right)$ for some $D_{i} \subseteq \mathcal{E}$, and

(c) $\sum_{A_{j} \in D_{i}}(2 p)^{t_{j}} \leq \frac{t_{i}}{2}$.

Then with positive probability, no event in $\mathcal{E}$ occurs.

Proof (of Lemma Q 2). Let $c \geq 4$ be a positive integer to be specified later. Let $p=\frac{1}{c}$. Then $0<p \leq \frac{1}{4}$. For each vertex $v \in V(G)$, randomly and independently choose $\operatorname{col}(v)$ from $\{1,2, \ldots, c\}$. For each edge $v w \in E(G)$, let $A_{v w}$ be the type-I event that $\operatorname{col}(v)=\operatorname{col}(w)$. Let $E^{\prime}$ be the set of $\operatorname{arcs} E^{\prime}=\{(v, w),(w, v): v w \in$ $E(G)\}$. For each pair of $\operatorname{arcs}(v, w),(x, y) \in E^{\prime}$ with no endpoint in common, let $B_{(v, w),(x, y)}$ be the type-II event that $\operatorname{col}(v)=\operatorname{col}(x)$ and $\operatorname{col}(w)=\operatorname{col}(y)$.

We will apply Lemma 3 to obtain a colouring such that no type-I event and no type-II event occurs. No type-I event implies that we have a (proper) vertex colouring. No type-II event implies that no two disjoint edges share the same pair of colours; that is, we have a strong star colouring. For each type-I event $A$, $\mathbf{P}(A)=\frac{1}{c}$. Let $t_{A}=1$. Then $\mathbf{P}(A)=p^{t_{A}}$. For each type-II event $B, \mathbf{P}(B)=\frac{1}{c^{2}}$. Let $t_{B}=2$. Then $\mathbf{P}(B)=p^{t_{B}}$. Thus condition (a) of Lemma 3 is satisfied.

An event involving a particular set of vertices is dependent only on other events involving at least one of the vertices in that set. Each vertex is involved in at most $\Delta$ type-I events, and at most $2 \Delta\left|E^{\prime}\right|=4 \Delta m$ type-II events. A type-I event involves two vertices, and is thus mutually independent of all but at most $2 \Delta$ type-I events and at most $8 \Delta m$ type-II events. A type-II event involves four vertices, and is thus mutually independent of all but at most $4 \Delta$ type-I events and at most $16 \Delta m$ type-II events. 
For condition (c) of Lemma 3 to hold we need $2 \Delta\left(\frac{2}{c}\right)^{1}+8 \Delta m\left(\frac{2}{c}\right)^{2} \leq \frac{1}{2}$ for the type-I events, and $4 \Delta\left(\frac{2}{c}\right)^{1}+16 \Delta m\left(\frac{2}{c}\right)^{2} \leq 1$ for the type-II events. It is a happy coincidence that these two equations are equivalent, and it is easily verified that $c=\lceil 4(\Delta+\sqrt{\Delta(1+4 m)})\rceil \geq 4$ is a solution. Thus by Lemma 3 with positive probability no type-I event and no type-II event occurs. Thus for every vertex $v \in V(G)$, there exists $\operatorname{col}(v) \in\{1, \ldots, c\}$ such that no type-I event and no typeII event occurs. As proved above such a colouring is a strong star colouring. Since $\Delta \leq \sqrt{\Delta m}$, the number of colours $c \leq\lceil 4(1+\sqrt{5}) \sqrt{\Delta m}\rceil \leq 14 \sqrt{\Delta m}$.

Theorems [1] and [2] imply:

Theorem 3. Every graph with $n$ vertices, $m$ edges and maximum degree $\Delta$ has ${ }_{a} \mathcal{O}\left((\Delta m)^{1 / 2}\right) \times \mathcal{O}\left((\Delta m)^{1 / 2}\right) \times \mathcal{O}(n)$ drawing with $\mathcal{O}(\Delta m n)$ volume.

We have the following corollary of Lemma 2.

Lemma 4. Every graph $G$ with $m$ edges has strong star chromatic number $\chi_{\mathrm{sst}}(G) \leq 15 \mathrm{~m}^{2 / 3}$.

Proof. Let $X$ be the set of vertices of $G$ with degree greater than $\frac{1}{4} m^{1 / 3}$. Let $H$ be the subgraph of $G$ induced by $V(G) \backslash X$. Thus $H$ has maximum degree at most $\frac{1}{4} m^{1 / 3}$. By Lemma 2, $H$ has a strong star colouring with at most $14\left(\frac{1}{4} m^{1 / 3} m\right)^{1 / 2}=7 m^{2 / 3}$ colours. Now $|X| \leq 2 m /\left(\frac{1}{4} m^{1 / 3}\right)=8 m^{2 / 3}$. By adding each vertex in $X$ to its own colour class we obtain a strong star colouring of $G$ with at most $15 \mathrm{~m}^{2 / 3}$ colours.

Since $\operatorname{tn}(G) \leq \chi_{\text {sst }}(G)$, Lemma 4 implies that $\operatorname{tn}(G) \leq 15 m^{2 / 3}$, and by Theorem 1 we have:

Theorem 4. Every graph with $n$ vertices and $m$ edges has a $\mathcal{O}\left(m^{2 / 3}\right) \times$ $\mathcal{O}\left(m^{2 / 3}\right) \times \mathcal{O}(n)$ drawing with $\mathcal{O}\left(m^{4 / 3} n\right)$ volume.

\section{Sub-quadratic Volume Bounds}

Vertex colourings 24] and track layouts [10] have previously been used to produce 3D drawings with small volume. In the following sequence of results we combine vertex colourings and track layouts to reduce the quadratic dependence on $t$ in Theorem 1 to linear. This comes at the expense of a higher dependence on the chromatic number. However, in the intended applications the chromatic number will be constant, or at least will be independent of the size of the graph. The proof of the next lemma is a further generalisation of the 'moment curve' method for producing three-dimensional graph drawings [52410].

Lemma 5. Let $G$ be a graph with a vertex c-colouring $\left\{V_{i}: 0 \leq i \leq c-1\right\}$, and a track layout $\left\{T_{i, j}: 0 \leq i \leq c-1,1 \leq j \leq t_{i}\right\}$, such that each $T_{i, j} \subseteq V_{i}$. Then $G$ has $a \mathcal{O}(c) \times \mathcal{O}\left(c^{2} t\right) \times \mathcal{O}\left(c^{5} t n^{\prime}\right)$ drawing, where $t=\max _{i} t_{i}$ and $n^{\prime}=\max _{i, j}\left|T_{i, j}\right|$. 
Proof. Let $p$ be the minimum prime such that $p \geq c$. Then $p<2 c$ by Bertrand's postulate. Let $v(i, j, k)$ denote the $k^{\text {th }}$ vertex in track $T_{i, j}$. Define

$$
\begin{aligned}
Y(i, j) & =p(2 i t+j)+\left(i^{2} \bmod p\right), \text { and } \\
Z(i, j, k) & =p\left(20 c i n^{\prime} \cdot Y(i, j)+k\right)+\left(i^{3} \bmod p\right) .
\end{aligned}
$$

Position each vertex $v(i, j, k)$ at the gridpoint $(i, Y(i, j), Z(i, j, k))$, and draw each edge as a line-segment between its endpoints. Since $Y(i, j) \in \mathcal{O}\left(c^{2} t\right)$ and $Z(i, j, k) \in \mathcal{O}\left(c^{3} n^{\prime} \cdot Y(i, j)\right)$, the drawing is $\mathcal{O}(c) \times \mathcal{O}\left(c^{2} t\right) \times \mathcal{O}\left(c^{5} t n^{\prime}\right)$.

Observe that the tracks from a single colour class are within a distinct $Y Z$ plane, each track occupies a distinct vertical line, and the $Z$-coordinates of the vertices within a track preserve the given ordering of that track. In addition, the $Y$-coordinates satisfy the following property.

Claim 1. For all distinct colours $i_{1}$ and $i_{2}$ and for all $1 \leq j_{1}, j_{2} \leq t$, we have $2 c \mid\left(Y\left(i_{1}, j_{1}\right)-Y\left(i_{2}, j_{2}\right) \mid\right.$ is greater than the $Y$-coordinate of any vertex.

Proof. Without loss of generality $i_{1}>i_{2}$. Observe that every $Y$-coordinate is less than $p(2(c-1) t+t)+p=p(2 c t-t+1) \leq 2 c p t$. Now $2 c\left|Y\left(i_{1}, j_{1}\right)-Y\left(i_{2}, j_{2}\right)\right|>$ $2 c\left|p\left(2 i_{1} t+1\right)-p\left(2 i_{2} t+t+1\right)\right| \geq 2 c p\left|2\left(i_{2}+1\right) t-\left(2 i_{2} t+t\right)\right|=2 c p t$.

We first prove that the only vertices each edge intersects are its own endpoints. It suffices to prove that if three tracks are collinear in the $X Y$-plane then they are all from the same colour class. Loosely speaking, an edge does not pass through any track. Clearly two tracks from the same colour class are not collinear (in the $X Y$-plane) with a third track from a distinct colour class. Thus we need only consider tracks $\left\{T\left(i_{\alpha}, j_{\alpha}\right): 1 \leq i_{\alpha} \leq 3\right\}$ from three distinct colour classes $\left\{i_{1}, i_{2}, i_{3}\right\}$. Let $R$ be the determinant,

$$
R=\left|\begin{array}{ccc}
1 & i_{1} & Y\left(i_{1}, j_{1}\right) \\
1 & i_{2} & Y\left(i_{2}, j_{2}\right) \\
1 & i_{3} & Y\left(i_{3}, j_{3}\right)
\end{array}\right|
$$

If the tracks $\left\{T\left(i_{\alpha}, j_{\alpha}\right): 1 \leq i_{\alpha} \leq 3\right\}$ are collinear in the $X Y$-plane then $R=0$. However $Y(i, j) \equiv i^{2}(\bmod p)$, and thus

$$
R \equiv\left|\begin{array}{ccc}
1 & i_{1} & i_{1}^{2} \\
1 & i_{2} & i_{2}^{2} \\
1 & i_{3} & i_{3}^{2}
\end{array}\right|=\prod_{1 \leq \alpha<\beta \leq 3}\left(i_{\alpha}-i_{\beta}\right) \not \equiv 0 \quad(\bmod p)
$$

since $i_{\alpha} \neq i_{\beta}$, and $p$ is a prime greater than any $i_{\alpha}-i_{\beta}$. Thus $R \neq 0$, and the tracks $\left\{T\left(i_{\alpha}, j_{\alpha}\right): 1 \leq i_{\alpha} \leq 3\right\}$ are not collinear in the $X Y$-plane. Hence the only vertices that an edge intersects are its own endpoints.

It remains to prove that there are no edge crossings. Consider two edges $e$ and $e^{\prime}$ with distinct endpoints $v\left(i_{\alpha}, j_{\alpha}, k_{\alpha}\right), 1 \leq \alpha \leq 4$. (Clearly edges with a common endpoint do not cross.) Let $Y_{\alpha}=Y\left(i_{\alpha}, j_{\alpha}\right)$. Consider the determinant

$$
D=\left|\begin{array}{cccc}
1 & i_{1} & Y_{1} & Z\left(i_{1}, j_{1}, k_{1}\right) \\
1 & i_{2} & Y_{2} & Z\left(i_{2}, j_{2}, k_{2}\right) \\
1 & i_{3} & Y_{3} & Z\left(i_{3}, j_{3}, k_{3}\right) \\
1 & i_{4} & Y_{4} & Z\left(i_{4}, j_{4}, k_{4}\right)
\end{array}\right| .
$$


If $e$ and $e^{\prime}$ cross then their endpoints are coplanar, and $D=0$. Thus it suffices to prove that $D \neq 0$. We proceed by considering the number $N=\left|\left\{i_{1}, i_{2}, i_{3}, i_{4}\right\}\right|$ of distinct colours assigned to the four endpoints of $e$ and $e^{\prime}$. Clearly $N \in\{2,3,4\}$.

Case $N=4$ : Since $Y_{\alpha} \equiv i_{\alpha}^{2}(\bmod p)$ and $Z\left(i_{\alpha}, j_{\alpha}, k_{\alpha}\right) \equiv i_{\alpha}^{3}(\bmod p)$,

$$
D \equiv\left|\begin{array}{cccc}
1 & i_{1} & i_{1}^{2} & i_{1}^{3} \\
1 & i_{2} & i_{2}^{2} & i_{2}^{3} \\
1 & i_{3} & i_{3}^{2} & i_{3}^{3} \\
1 & i_{4} & i_{4}^{2} & i_{4}^{3}
\end{array}\right|=\prod_{1 \leq \alpha<\beta \leq 4}\left(i_{\alpha}-i_{\beta}\right) \not \equiv 0 \quad(\bmod p)
$$

since $i_{\alpha} \neq i_{\beta}$, and $p$ is a prime greater than any $i_{\alpha}-i_{\beta}$. Thus $D \neq 0$.

Case $N=3$ : Without loss of generality $i_{1}=i_{2}$. It follows that $D=5 S_{0}+$ $S_{1}+S_{2}+S_{3}+S_{4}$ where

$S_{0}=4 c p n^{\prime}\left(i_{3}-i_{1}\right)\left(i_{4}-i_{1}\right)\left(Y_{2}-Y_{1}\right)\left(Y_{3}-Y_{4}\right)$

$S_{1}=p\left(Y_{2}-Y_{1}\right)\left(k_{3}\left(i_{4}-i_{1}\right)-k_{4}\left(i_{3}-i_{1}\right)\right)$

$S_{2}=p\left(i_{4}-i_{3}\right)\left(k_{2} Y_{1}-k_{1} Y_{2}\right)$

$S_{3}=p\left(k_{2}-k_{1}\right)\left(Y_{4}\left(i_{3}-i_{1}\right)-Y_{3}\left(i_{4}-i_{1}\right)\right)$

$S_{4}=\left(Y_{2}-Y_{1}\right)\left(\left(i_{3}-i_{4}\right)\left(i_{1}^{3} \bmod p\right)-\left(i_{3}-i_{1}\right)\left(i_{4}^{3} \bmod p\right)+\left(i_{4}-i_{1}\right)\left(i_{3}^{3} \bmod p\right)\right)$.

If $Y_{1}=Y_{2}$ then $e$ and $e^{\prime}$ do not cross, since no three tracks from distinct colour classes are collinear in the $X Y$-plane. Assume $Y_{1} \neq Y_{2}$. If $i_{3}<i_{1}<i_{4}$ or $i_{4}<i_{1}<i_{3}$ then $e$ and $e^{\prime}$ do not cross, simply by considering the projection in the $X Y$-plane. Thus $i_{1}<i_{3}, i_{4}$ or $i_{1}>i_{3}, i_{4}$, which implies

$$
\left(i_{4}-i_{1}\right)\left(i_{3}-i_{1}\right)>\left|i_{4}-i_{3}\right| \text {. }
$$

Claim 2. If $\left|S_{0}\right| \geq\left|S_{1}\right|,\left|S_{0}\right| \geq\left|S_{2}\right|,\left|S_{0}\right| \geq\left|S_{3}\right|$ and $\left|S_{0}\right| \geq\left|S_{4}\right|$ then $D \neq 0$.

Proof. To prove that $D=5 S_{0}+S_{1}+S_{2}+S_{3}+S_{4}$ is nonzero it suffices to show that $D^{\prime}= \pm 5\left|S_{0}\right| \pm\left|S_{1}\right| \pm\left|S_{2}\right| \pm\left|S_{3}\right| \pm\left|S_{4}\right|$ is nonzero for all combinations of pluses and minuses. Consider $X= \pm\left|S_{1}\right| \pm\left|S_{2}\right| \pm\left|S_{3}\right| \pm\left|S_{4}\right|$ for some combination of pluses and minuses. Since $\left|S_{1}\right| \leq\left|S_{0}\right|,\left|S_{2}\right| \leq\left|S_{0}\right|,\left|S_{3}\right| \leq\left|S_{0}\right|$, and $\left|S_{4}\right| \leq\left|S_{0}\right|$, we have $-4\left|S_{0}\right| \leq X \leq 4\left|S_{0}\right|$. Since $S_{0} \neq 0$, we have $5\left|S_{0}\right|+X \neq 0$ and $-5\left|S_{0}\right|+X \neq 0$. That is, all values of $D^{\prime}$ are nonzero. Therefore $D \neq 0$.

Therefore, to prove that $D \neq 0$ it suffices to show that $\left|S_{0}\right| \geq\left|S_{1}\right|,\left|S_{0}\right| \geq$ $\left|S_{2}\right|,\left|S_{0}\right| \geq\left|S_{3}\right|$ and $\left|S_{0}\right| \geq\left|S_{4}\right|$. We will use the following elementary facts regarding absolute values:

$$
\begin{aligned}
& \forall a_{1}, \ldots, a_{k} \in \mathbb{R} \quad\left|a_{1} a_{2} \ldots a_{k}\right|=\left|a_{1}\right|\left|a_{2}\right| \cdots\left|a_{k}\right|, \text { and } \\
& \left|a_{1}+a_{2}+\cdots+a_{k}\right| \leq\left|a_{1}\right|+\left|a_{2}\right|+\cdots+\left|a_{k}\right| \leq k \cdot \max \left\{\left|a_{1}\right|,\left|a_{2}\right|, \ldots,\left|a_{k}\right|\right\} .
\end{aligned}
$$

- First we prove that $\left|S_{0}\right| \geq\left|S_{1}\right|$. That is,

$\left|4 c p n^{\prime}\left(i_{3}-i_{1}\right)\left(i_{4}-i_{1}\right)\left(Y_{2}-Y_{1}\right)\left(Y_{3}-Y_{4}\right)\right| \geq\left|p\left(Y_{2}-Y_{1}\right)\left(k_{3}\left(i_{4}-i_{1}\right)-k_{4}\left(i_{3}-i_{1}\right)\right)\right|$. 
Hence, $\left|S_{0}\right|>\left|S_{1}\right|$ is implied if

$$
\begin{aligned}
& 2 n^{\prime}\left|i_{3}-i_{1}\right|\left|i_{4}-i_{1}\right|\left|Y_{3}-Y_{4}\right| \geq\left|k_{3}\left(i_{4}-i_{1}\right)-k_{4}\left(i_{3}-i_{1}\right)\right| . \\
& \Longleftarrow 2 n^{\prime}\left|i_{3}-i_{1}\right|\left|i_{4}-i_{1}\right|\left|Y_{3}-Y_{4}\right| \geq 2 \cdot \max \left\{\left|k_{4}\left(i_{3}-i_{1}\right)\right|,\left|k_{3}\left(i_{4}-i_{1}\right)\right|\right\} .
\end{aligned}
$$

Since $n^{\prime} \geq k_{3}, k_{4}$ and $\left|Y_{3}-Y_{4}\right| \geq 1$,

$$
\left|S_{0}\right|>\left|S_{1}\right| \Longleftarrow\left|i_{3}-i_{1}\right|\left|i_{4}-i_{1}\right| \geq \max \left\{\left|i_{3}-i_{1}\right|,\left|i_{4}-i_{1}\right|\right\} .
$$

Thus $\left|S_{0}\right| \geq\left|S_{1}\right|$ since $\left|i_{3}-i_{1}\right| \geq 1$ and $\left|i_{4}-i_{1}\right| \geq 1$.

- Now we prove that $\left|S_{0}\right| \geq\left|S_{2}\right|$. That is,

$$
\left|4 c p n^{\prime}\left(i_{3}-i_{1}\right)\left(i_{4}-i_{1}\right)\left(Y_{2}-Y_{1}\right)\left(Y_{3}-Y_{4}\right)\right| \geq\left|p\left(i_{4}-i_{3}\right)\left(k_{2} Y_{1}-k_{1} Y_{2}\right)\right| .
$$

By (11) and since $\left|Y_{2}-Y_{1}\right| \geq 1,\left|S_{0}\right| \geq\left|S_{2}\right|$ is implied if $\left|4 c n^{\prime}\left(Y_{3}-Y_{4}\right)\right| \geq$ $\left|k_{2} Y_{1}-k_{1} Y_{2}\right|$. This in turn is implied if $\left|2 c n^{\prime}\left(Y_{3}-Y_{4}\right)\right| \geq \max \left\{\left|k_{2} Y_{1}\right|,\left|k_{1} Y_{2}\right|\right\}$, which holds since $n^{\prime} \geq k_{1}, k_{2}$ and $\left|2 c\left(Y_{3}-Y_{4}\right)\right| \geq \max \left\{Y_{1}, Y_{2}\right\}$ by Claim 1 .

- Now we prove that $\left|S_{0}\right| \geq\left|S_{3}\right|$. That is,

$\left|4 c p n^{\prime}\left(i_{3}-i_{1}\right)\left(i_{4}-i_{1}\right)\left(Y_{2}-Y_{1}\right)\left(Y_{3}-Y_{4}\right)\right| \geq\left|p\left(k_{2}-k_{1}\right)\left(Y_{4}\left(i_{3}-i_{1}\right)-Y_{3}\left(i_{4}-i_{1}\right)\right)\right|$.

Now $n^{\prime} \geq\left|k_{2}-k_{1}\right|$ and $\left|Y_{2}-Y_{1}\right| \geq 1$. Thus $\left|S_{0}\right| \geq\left|S_{3}\right|$ is implied if

$$
\begin{aligned}
\left|4 c\left(i_{3}-i_{1}\right)\left(i_{4}-i_{1}\right)\left(Y_{3}-Y_{4}\right)\right| & \geq\left|Y_{4}\left(i_{3}-i_{1}\right)-Y_{3}\left(i_{4}-i_{1}\right)\right| . \\
\Longleftarrow\left|2 c\left(i_{3}-i_{1}\right)\left(i_{4}-i_{1}\right)\left(Y_{3}-Y_{4}\right)\right| & \geq \max \left\{\left|Y_{4}\left(i_{3}-i_{1}\right)\right|,\left|Y_{3}\left(i_{4}-i_{1}\right)\right|\right\},
\end{aligned}
$$

which holds since $\left|2 c\left(Y_{3}-Y_{4}\right)\right| \geq \max \left\{Y_{1}, Y_{2}\right\}$ by Claim 1 .

- Finally we prove that $\left|S_{0}\right| \geq\left|S_{4}\right|$. That is,

$$
\begin{aligned}
& \left|4 c p n^{\prime}\left(i_{3}-i_{1}\right)\left(i_{4}-i_{1}\right)\left(Y_{2}-Y_{1}\right)\left(Y_{3}-Y_{4}\right)\right| \geq \\
& \quad\left|\left(Y_{2}-Y_{1}\right)\left(\left(i_{3}-i_{4}\right)\left(i_{1}^{3} \bmod p\right)-\left(i_{3}-i_{1}\right)\left(i_{4}^{3} \bmod p\right)+\left(i_{4}-i_{1}\right)\left(i_{3}^{3} \bmod p\right)\right)\right| .
\end{aligned}
$$

Now $c n^{\prime}\left|Y_{3}-Y_{4}\right| \geq 1$. Thus $\left|S_{0}\right|>\left|S_{4}\right|$ is implied if

$$
\begin{aligned}
& \left|3 p\left(i_{3}-i_{1}\right)\left(i_{4}-i_{1}\right)\right| \geq \\
& \left|\left(i_{3}-i_{4}\right)\left(i_{1}^{3} \bmod p\right)-\left(i_{3}-i_{1}\right)\left(i_{4}^{3} \bmod p\right)+\left(i_{4}-i_{1}\right)\left(i_{3}^{3} \bmod p\right)\right| \\
\Longleftarrow & \left|3 p\left(i_{3}-i_{1}\right)\left(i_{4}-i_{1}\right)\right| \geq \\
& 3 \cdot \max \left\{\left|\left(i_{3}-i_{4}\right)\left(i_{1}^{3} \bmod p\right)\right|,\left|\left(i_{3}-i_{1}\right)\left(i_{4}^{3} \bmod p\right)\right|,\left|\left(i_{4}-i_{1}\right)\left(i_{3}^{3} \bmod p\right)\right|\right\} \\
\Longleftarrow & \left|\left(i_{3}-i_{1}\right)\left(i_{4}-i_{1}\right)\right| \geq \max \left\{\left|i_{3}-i_{4}\right|,\left|i_{3}-i_{1}\right|,\left|i_{4}-i_{1}\right|\right\},
\end{aligned}
$$

which holds by (11).

Case $N=2$ : Without loss of generality $i_{1}=i_{2} \neq i_{3}=i_{4}$. If $Y_{1}=Y_{2}$ and $Y_{3}=Y_{4}$ then $e$ and $e^{\prime}$ do not cross as otherwise there would be an X-crossing in the track layout. If $Y_{1}=Y_{2}$ and $Y_{3} \neq Y_{4}\left(\right.$ or $Y_{1} \neq Y_{2}$ and $\left.Y_{3}=Y_{4}\right)$ then $e$ 
and $e^{\prime}$ do not cross, by considering the projection in the $X Y$-plane. Thus we can assume that $Y_{1} \neq Y_{2}$ and $Y_{3} \neq Y_{4}$. It follows that

$$
\begin{aligned}
D=p\left(i_{1}-i_{3}\right)( & 5 \cdot 4 c n^{\prime}\left(Y_{2}-Y_{1}\right)\left(Y_{4}-Y_{3}\right)\left(i_{3}-i_{1}\right) \\
& \left.+\left(k_{1}-k_{2}\right)\left(Y_{4}-Y_{3}\right)+\left(k_{4}-k_{3}\right)\left(Y_{2}-Y_{1}\right)\right) .
\end{aligned}
$$

As in Claim 2, to show that $D \neq 0$ it suffices to show that

$$
\begin{aligned}
\left|4 c n^{\prime}\left(Y_{2}-Y_{1}\right)\left(Y_{4}-Y_{3}\right)\left(i_{3}-i_{1}\right)\right| & \geq\left|\left(k_{1}-k_{2}\right)\left(Y_{4}-Y_{3}\right)\right|, \\
\text { and }\left|4 c n^{\prime}\left(Y_{2}-Y_{1}\right)\left(Y_{4}-Y_{3}\right)\left(i_{3}-i_{1}\right)\right| & \geq\left|\left(k_{4}-k_{3}\right)\left(Y_{2}-Y_{1}\right)\right| .
\end{aligned}
$$

Inequalities (21) and (3) hold since $n^{\prime}>\left|k_{1}-k_{2}\right|$ and $n^{\prime}>\left|k_{4}-k_{3}\right|$.

Note that the constant 20 in the definition of $Z(i, j, k)$ in the proof of Lemma 5 is chosen to enable a relatively simple proof. It is easily seen that it can be reduced. The proof of the next lemma is based on an idea of Pach et al. 24] for balancing the size of the colour classes in a vertex colouring.

Lemma 6. Let $G$ be an n-vertex graph with a c-colouring $\left\{V_{i}: 0 \leq i \leq c-1\right\}$ and a track layout $\left\{T_{i, j}: 0 \leq i \leq c-1,1 \leq j \leq t_{i}\right\}$, such that each $T_{i, j} \subseteq V_{i}$. Let $k=\sum_{i} t_{i}$ be the total number of tracks. Then $G$ has a $\mathcal{O}(c) \times \mathcal{O}(c k) \times \mathcal{O}\left(c^{4} n\right)$ drawing.

Proof. Replace each track by tracks of size exactly $\left\lceil\frac{n}{k}\right\rceil$, except for at most one track of size at most $\left\lceil\frac{n}{k}\right\rceil$. Order the vertices within each track according to the original track, and consider the new tracks to belong to the same colour class as the original. Clearly no X-crossing is created. Within $V_{i}$ there are now at most $t_{i}+\left|V_{i}\right| /\left\lceil\frac{n}{k}\right\rceil$ tracks. The total number of tracks is $\sum_{i}\left(t_{i}+\left|V_{i}\right| /\left\lceil\frac{n}{k}\right\rceil\right) \leq 2 k$. For each colour class $V_{i}$, partition the set of tracks in $V_{i}$ into sets of size exactly $\left\lceil\frac{2 k}{c}\right\rceil$, except for one set of size at most $\left\lceil\frac{2 k}{c}\right\rceil$. Consider each set to correspond to a colour. The number of colours is now at most $c+2 k /\left\lceil\frac{2 k}{c}\right\rceil \leq 2 c$. Applying Lemma 5 with $2 c$ colours, $n^{\prime}=\left\lceil\frac{n}{k}\right\rceil$, and $t=\left\lceil\frac{2 k}{c}\right\rceil$, we obtain the desired drawing.

Theorem 5. Every c-colourable graph $G$ with $n$ vertices and track-number $\operatorname{tn}(G) \leq t$ has a $\mathcal{O}(c) \times \mathcal{O}\left(c^{2} t\right) \times \mathcal{O}\left(c^{4} n\right)$ drawing with $\mathcal{O}\left(c^{7}\right.$ tn $)$ volume.

Proof. Let $\left\{V_{i}: 0 \leq i \leq c-1\right\}$ be a $c$-colouring of $G$. Let $\left\{T_{j}: 1 \leq j \leq t\right\}$ be a $t$-track layout of $G$. For all $0 \leq i \leq c-1$ and $1 \leq j \leq t$, let $T_{i, j}=V_{i} \cap T_{j}$. Then $\left\{V_{i}: 0 \leq i \leq c-1\right\}$ and $\left\{T_{i, j}: 0 \leq i \leq c-1,1 \leq j \leq t\right\}$ satisfy Lemma $[6]$ with $k=c t$. Thus $G$ has the desired drawing.

In the case of bipartite graphs we have a simple proof of Theorem 5 with improved constants.

Lemma 7. Every $n$-vertex bipartite graph $G$ with track-number $\operatorname{tn}(G) \leq t$ has a $2 \times t \times n$ drawing. 
Proof. Let $\{A, B\}$ be the bipartition of $V(G)$. Let $\left\{T_{i}: 1 \leq i \leq t\right\}$ be a $t$-track layout of $G$. For each $1 \leq i \leq t$, let $A_{i}=T_{i} \cap A$ and $B_{i}=T_{i} \cap B$. Order each $A_{i}$ and $B_{i}$ as in $T_{i}$. Place the $j^{\text {th }}$ vertex in $A_{i}$ at $\left(0, i, j+\sum_{k=1}^{i-1}\left|A_{k}\right|\right)$. Place the $j^{\text {th }}$ vertex in $B_{i}$ at $\left(1, t-i+1, j+\sum_{k=1}^{i-1}\left|B_{k}\right|\right)$. The drawing is thus $2 \times t \times n$. Let $A_{i} B_{j}$ be the set of edges with one endpoint in $A_{i}$ and the other in $B_{j}$. There is no crossing between edges in $A_{i} B_{j}$ and $A_{i} B_{j}$ as otherwise there would be an $\mathrm{X}$-crossing in the track layout. Clearly there is no crossing between edges in $A_{i} B_{j}$ and $A_{i} B_{k}$ for $j \neq k$. Suppose there is a crossing between edges in $A_{i} B_{j}$ and $A_{k} B_{\ell}$ with $i \neq k$ and $j \neq \ell$. Without loss of generality $i<k$. Then the projections of the edges in the $X Y$-plane also cross, and thus $j<\ell$. Hence the projections in the $X Z$-plane do not cross, and thus the edges do not cross.

Lemma 4 with $\operatorname{tn}(G) \leq \chi_{\text {sst }}(G)$ and Theorem 5 imply:

Theorem 6. Every c-colourable graph with $n$ vertices and $m$ edges has a $\mathcal{O}(c) \times$ $\mathcal{O}\left(c^{2} m^{2 / 3}\right) \times \mathcal{O}\left(c^{4} n\right)$ drawing with $\mathcal{O}\left(c^{6} m^{2 / 3} n\right)$ volume.

The next result is one of the main contributions of this paper.

Theorem 7. Every planar graph with $n$ vertices has a $\mathcal{O}(1) \times \mathcal{O}\left(n^{1 / 2}\right) \times \mathcal{O}(n)$ drawing with $\mathcal{O}\left(n^{3 / 2}\right)$ volume.

Proof. Planar graphs have $\mathcal{O}\left(n^{1 / 2}\right)$ path-width (see [2]), and thus have $\mathcal{O}\left(n^{1 / 2}\right)$ track-number by Lemma 1(a). The result follows from Theorem 5 since planar graphs are 4-colourable.

The following generalisation of Theorem 7 follows from Lemma 1 (d), Theorem [5] and the classical result of Heawood [20] that $\chi(G) \in \mathcal{O}\left(\gamma^{1 / 2}\right)$.

Theorem 8. Every $n$-vertex graph with genus $\gamma$ has a $\mathcal{O}\left(\gamma^{1 / 2}\right) \times \mathcal{O}\left(\gamma^{3 / 2} n^{1 / 2}\right) \times$ $\mathcal{O}\left(\gamma^{2} n\right)$ drawing with $\mathcal{O}\left(\gamma^{4} n^{3 / 2}\right)$ volume.

The next generalisation of Theorem 7 for graphs with no $K_{h}$-minor follows from Lemma 1(e), Theorem 5 and the result independently due to Kostochka [21] and Thomason [26] that $\chi(G) \in \mathcal{O}\left(h \log ^{1 / 2} h\right)$.

Theorem 9. Every $n$-vertex graph with no $K_{h}$-minor has a $\mathcal{O}\left(h \log ^{1 / 2} h\right) \times$ $\mathcal{O}\left(h^{7 / 2} \log h \cdot n^{1 / 2}\right) \times \mathcal{O}\left(h^{4} \log ^{2} h \cdot n\right)$ drawing with volume $\mathcal{O}\left(h^{17 / 2} \log ^{7 / 2} h \cdot n^{3 / 2}\right)$.

Finally we consider the maximum degree $\Delta$ as a parameter. By the sequential greedy algorithm, $G$ is $(\Delta+1)$-colourable. Thus by Theorems 2 and 5 we have:

Theorem 10. Every graph with $n$ vertices, $m$ edges, and maximum degree $\Delta$ has a $\mathcal{O}(\Delta) \times \mathcal{O}\left(\Delta^{5 / 2} m^{1 / 2}\right) \times \mathcal{O}\left(\Delta^{4} n\right)$ drawing with $\mathcal{O}\left(\Delta^{15 / 2} m^{1 / 2} n\right)$ volume.

Graphs with constant $\Delta$ have $\mathcal{O}(n)$ edges. By Theorems 8 , 9 and 10 we have:

Corollary 1. Every $n$-vertex graph with constant genus, or with no $K_{h}$-minor for some constant $h$, or with constant maximum degree has a $\mathcal{O}(1) \times \mathcal{O}\left(n^{1 / 2}\right) \times$ $\mathcal{O}(n)$ drawing with $\mathcal{O}\left(n^{3 / 2}\right)$ volume. 
We conclude with the following open problems: Does every graph have a $3 \mathrm{D}$ drawing with $\mathcal{O}(\mathrm{nm})$ volume? Does every graph with constant chromatic number have a 3D drawing with $\mathcal{O}(n \sqrt{m})$ volume? These bounds match the lower bounds for $K_{n}$ and $K_{n, n}$, and would make edge-sensitive improvements to the existing upper bounds of $\mathcal{O}\left(n^{3}\right)$ and $\mathcal{O}\left(n^{2}\right)$, respectively. These edge-sensitive bounds would be implied by Theorems 1 and 5 should every graph have $\mathcal{O}(\sqrt{m})$ track-number. In turn, this bound on track-number would be implied should every graph have $\mathcal{O}(\sqrt{m})$ strong star chromatic number. As far as the authors are aware, a $\mathcal{O}(\sqrt{m})$ bound is not even known for star chromatic number. The best known bound in this direction is $\chi_{\mathrm{st}}(G) \leq 11 \mathrm{~m}^{3 / 5}$, which can be proved in a similar fashion to Lemma 4 in conjunction with the result of Fertin et al. [15] that $\chi_{\text {st }}(G) \leq\left\lceil 20 \Delta^{3 / 2}\right\rceil$ (see [1]).

Acknowledgements. Thanks to Stefan Langerman for stimulating discussions. Thanks to Ferran Hurtado and Prosenjit Bose for graciously hosting the second author, whose research was completed at the Departament de Matemàtica Aplicada II, Universitat Politècnica de Catalunya, Barcelona, Spain.

\section{References}

1. Noga Alon, Paul Seymour, and Robin Thomas. A separator theorem for nonplanar graphs. J. Amer. Math. Soc., 3(4):801-808, 1990.

2. HANS L. BodlaEnder. A partial $k$-arboretum of graphs with bounded treewidth. Theoret. Comput. Sci., 209(1-2):1-45, 1998.

3. Prosenjit Bose, Jurek Czyzowicz, Pat Morin, and David R. Wood. The maximum number of edges in a three-dimensional grid-drawing. In Proc. 19th European Workshop on Computational Geometry, pages 101-103, Germany, 2003. Univ. of Bonn.

4. Tiziana Calamoneri and Andrea Sterbini. 3D straight-line grid drawing of 4-colorable graphs. Inform. Process. Lett., 63(2):97-102, 1997.

5. Robert F. Cohen, Peter Eades, Tao Lin, and Frank Ruskey. Threedimensional graph drawing. Algorithmica, 17(2):199-208, 1996.

6. Emilio Di Giacomo. Drawing series-parallel graphs on restricted integer 3D grids. In these proceedings.

7. Emilio Di Giacomo, Giuseppe Liotta, and Stephen Wismath. Drawing seriesparallel graphs on a box. In Proc. 14th Canadian Conf. on Computational Geometry (CCCG '02), pages 149-153. The Univ. of Lethbridge, Canada, 2002.

8. Emilio Di Giacomo and Henk MeiJer. Track drawings of graphs with constant queue number. In these proceedings.

9. Hristo N. Djidjev. A separator theorem. C. R. Acad. Bulgare Sci., 34(5):643645, 1981.

10. Vida Dujmović, Pat Morin, and David R. Wood. Path-width and threedimensional straight-line grid drawings of graphs. In Michael T. Goodrich and Stephen G. Kobourov, eds., Proc. 10th Int'l Symp. on Graph Drawing (GD '02), volume 2528 of Lecture Notes in Comput. Sci., pages 42-53. Springer, 2002.

11. Vida Dujmović and David R. Wood. New results in graph layout. Tech. Report TR-2003-04, School of Computer Science, Carleton Univ., Ottawa, Canada, 2003. 
12. Vida Dujmović and David R. Wood. Tree-partitions of $k$-trees with applications in graph layout. In Hans L. Bodlaender, ed., Proc. 29th Workshop on Graph Theoretic Concepts in Comput. Sci. (WG'03), Lecture Notes in Comput. Sci. Springer, to appear.

13. PAUl ERdős and LÁszló Lovász. Problems and results on 3-chromatic hypergraphs and some related questions. In Infinite and Finite Sets, volume 10 of Colloq. Math. Soc. János Bolyai, pages 609-627. North-Holland, 1975.

14. Stefan Felsner, Giussepe Liotta, and Stephen Wismath. Straight-line drawings on restricted integer grids in two and three dimensions. In PETRA Mutzel, Michael Jünger, and Sebastian Leipert, eds., Proc. 9th Int'l Symp. on Graph Drawing (GD '01), volume 2265 of Lecture Notes in Comput. Sci., pages 328-342. Springer, 2002.

15. Guillaume Fertin, André Raspaud, and Bruce Reed. On star coloring of graphs. In Andreas Branstädt and VAn Bang Le, eds., Proc. 27th Int'l Workshop on Graph-Theoretic Concepts in Computer Science (WG '01), volume 2204 of Lecture Notes in Comput. Sci., pages 140-153. Springer, 2001.

16. John R. Gilbert, Joan P. Hutchinson, and Robert E. TARjan. A separator theorem for graphs of bounded genus. J. Algorithms, 5(3):391-407, 1984.

17. András Gyárfás and Douglas West. Multitrack interval graphs. In Proc. 26th Southeastern Int'l Conf. on Combinatorics, Graph Theory and Computing, volume 109 of Congr. Numer., pages 109-116, 1995.

18. Toru Hasunuma. Laying out iterated line digraphs using queues. In these proceedings.

19. Lenwood S. Heath, Frank Thomson Leighton, and Arnold L. Rosenberg. Comparing queues and stacks as mechanisms for laying out graphs. SIAM J. Discrete Math., 5(3):398-412, 1992.

20. Percy J. Heawood. Map colour theorem. Quart. J. Pure Appl. Math., 24:332338, 1890.

21. Alexandr V. Kostochka. The minimum Hadwiger number for graphs with a given mean degree of vertices. Metody Diskret. Analiz., 38:37-58, 1982.

22. Michael Molloy and BRuce ReED. Graph colouring and the probabilistic method, volume 23 of Algorithms and Combinatorics. Springer, 2002.

23. Jaroslav NešetřIl and Patrice Ossona de Mendez. Colorings and homomorphisms of minor closed classes. In Boris Aronov, Saugata Basu, János Pach, and Micha Sharir, eds., Discrete and Computational Geometry, The GoodmanPollack Festschrift, volume 25 of Algorithms and Combinatorics. Springer, 2003.

24. János Pach, Torsten Thiele, and GÉza Tóth. Three-dimensional grid drawings of graphs. In Giuseppe Di Battista, ed., Proc. 5th Int'l Symp. on Graph Drawing (GD '97), volume 1353 of Lecture Notes in Comput. Sci., pages 47-51. Springer, 1997. Also in Bernard Chazelle, Jacob E. Goodman, and Richard Pollack, eds., Advances in discrete and computational geometry, volume 223 of Contempory Mathematics, pp. 251-255, Amer. Math. Soc., 1999.

25. Timo Poranen. A new algorithm for drawing series-parallel digraphs in 3D. Tech. Report A-2000-16, Dept. of Computer and Information Sciences, Univ. of Tampere, Finland, 2000.

26. Andrew Thomason. An extremal function for contractions of graphs. Math. Proc. Cambridge Philos. Soc., 95(2):261-265, 1984.

27. David R. Wood. Queue layouts, tree-width, and three-dimensional graph drawing. In Manindra Agrawal and Anil Seth, eds., Proc. 22nd Foundations of Software Technology and Theoretical Computer Science (FST TCS '02), volume 2556 of Lecture Notes in Comput. Sci., pages 348-359. Springer, 2002. 